\title{
Point of Care Ultrasound Use by Interns in Emergency Department
}

\author{
Kamonwon lenghong ${ }^{1}$, Takaaki Suzuki ${ }^{2}$, Somsak Tiamkao ${ }^{3}$, Dhanu Gaysonsiri ${ }^{4}$, Vajarabhongsa Bhudhisawasdi ${ }^{1}$, \\ Korakot Apiratwarakul ${ }^{1 *}$ ii \\ ${ }^{1}$ Department of Emergency Medicine, Faculty of Medicine, Khon Kaen University, Khon Kaen, Thailand; ${ }^{2}$ Department of \\ Emergency and Critical Care Medicine, University of Tsukuba Hospital, Tsukuba, Japan; ${ }^{3}$ Department of Medicine, Faculty \\ of Medicine, Khon Kaen University, Khon Kaen, Thailand; ${ }^{4}$ Department of Pharmacology, Faculty of Medicine, Khon Kaen \\ University, Khon Kaen, Thailand
}

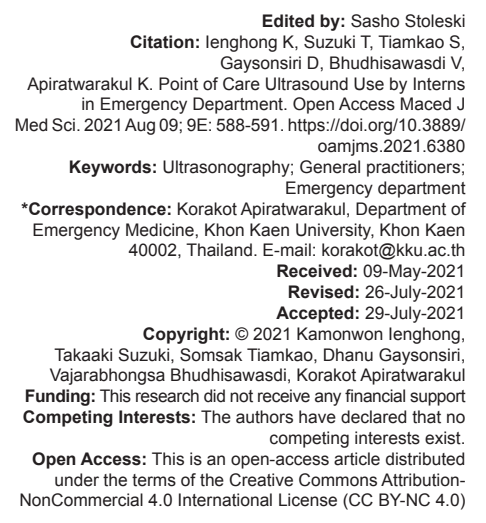

\section{Introduction}

Point-of-Care Ultrasound (POCUS) is an essential tool used to aid physicians in getting an accurate diagnosis and can often be indispensable when performing procedures [1]. Hence, POCUS is increasingly being used by physicians working in emergency departments including emergency physicians, emergency medicine residents, and interns [2], [3], [4].

Interns work in a range of settings and levels of various degrees of urgency, from general practice services as general practitioners in primary care to the undifferentiated emergency medicine. However, most of the emergency services in Thailand are being worked by internists who have had less ultrasound experience than might previously have been thought.

The use of ultrasonography is userdependent. Previous studies [5] have demonstrated that general practitioners performing point-of-care ultrasound examinations had a very high rate of interrater agreement when compared with specialists.
Moreover, the frequency of ultrasonography was quite high in a general practice setting for broad applications [6], [7], [8], [9].

Nevertheless, the research on POCUS performed by internists in Thailand is still rather limited. In this study, we aimed to explore the number of ultrasound examinations performed by interns in the emergency department, identify the characteristics of POCUS use, and the barriers to performing POCUS examinations faced by interns.

\section{Methods}

\section{Study design}

This was a retrospective, single-centered, observational study at a tertiary university hospital in Khon Kaen, Thailand. Ethical approval was provided by the Khon Kaen University Ethics Committee for Human Research (HE641238). 


\section{Sample size}

The interns, who were in the emergency medicine rotation at the Department of Emergency Medicine at Khon Kaen University's Srinagarind Hospital from July 2020 to April 2021, were enrolled in this study. The interns, who had not participated in this rotation, were excluded from the study. Hence, based on a questionnaire study [10], we estimate that there were around eighty interns who would meet our inclusion criteria. Assuming a participation rate of $49 \%$ [10], we expected to include at least forty interns in this study.

\section{Study protocol}

At the end of their rotation, they took a selfadministered questionnaire using a 5-point Likert scale via Google Forms. An email containing a link to the survey was sent to each participant. The survey consisted of seven questions about participants' experiences performing POCUS examinations (Appendix 1). The survey and collected data were hosted by the emergency medicine department. To ensure anonymity, yet allow contact with non-responders, each participant was given access to a personalized but de-identified online survey. The primary outcome of this study was to identify the frequency and collect feedback on administering POCUS examinations from the interns in this rotation.

\section{Statistical analysis}

Mean Likert scale values for each electronic survey response were used to represent overall participant agreement. Participant perceptions were presented as frequencies with percentages. Responses were summarized into five categories to reflect agreement: Very satisfied (5), satisfied (4), neutral (3), dissatisfied (2), and very dissatisfied (1). All data analyses were performed using Stata version 10.1 (StataCorp, College Station, TX).

\section{Results}

From July 2020 to April 2021 responses were obtained from $81.25 \%(65 / 80)$, the majority of respondents were mainly female $(60 \%)$ and the average age was 26 .

The average number of POCUS examinations per physician per shift was 2.5 POCUS examinations. Each intern performed between 10 and 72 POCUS examinations in this rotation. POCUS examinations were performed mostly in the morning shift (79\%) with use for diagnostic purposes only (100\%) and no procedure-related purposes. The large majority of POCUS usage rates $(80 \%)$ were found performing abdominal examinations. The most commonly used POCUS application was FAST (Focused Assessment with Sonography for Trauma) examination (92.3\%) (Table 1).

Table 1: The characteristic of POCUS use by interns

\begin{tabular}{ll}
\hline Items & Frequency (\%) \\
\hline The average number of POCUS examinations per physician per shift & 2.5 \\
The total POCUS examinations per physician per rotation & $10-72$ \\
Time of POCUS examination $(\mathrm{n}=65)$ & \\
$\quad$ Morning shift & $51(78.46)$ \\
$\quad$ Afternoon shift & $10(15.38)$ \\
$\quad$ Evening shift & $4(6.15)$ \\
Purpose of POCUS examination ( $\mathrm{n}=65)$ & $65(100)$ \\
$\quad$ Diagnostic & \\
The area of POCUS examination & $52(80)$ \\
Abdomen & $48(31.2)$ \\
KUB system & $44(28.6)$ \\
Inferior vena cava & $25(16.25)$ \\
Heart & $20(13)$ \\
Lungs & $14(9.1)$ \\
OB-GYN & $60(92.3)$ \\
The POCUS protocol & 0 \\
FAST (the Focused assessment with sonography in trauma) & 0 \\
CASA (the Cardiac Arrest Sonographic Assessment) & 0 \\
BLUE (the Bedside lung ultrasound in emergency) & \\
RUSH (the Rapid Ultrasound for Shock and Hypotension) & \\
\hline
\end{tabular}

Most respondents (65\%) rated their confidence level to perform POCUS at a 3 . The most commonly reported barriers to performing POCUS were lack of POCUS training $(47 \%)$, the emergency department overcrowding (30\%), and insufficient access to a POCUS machine (20.5\%) (Figure 1).

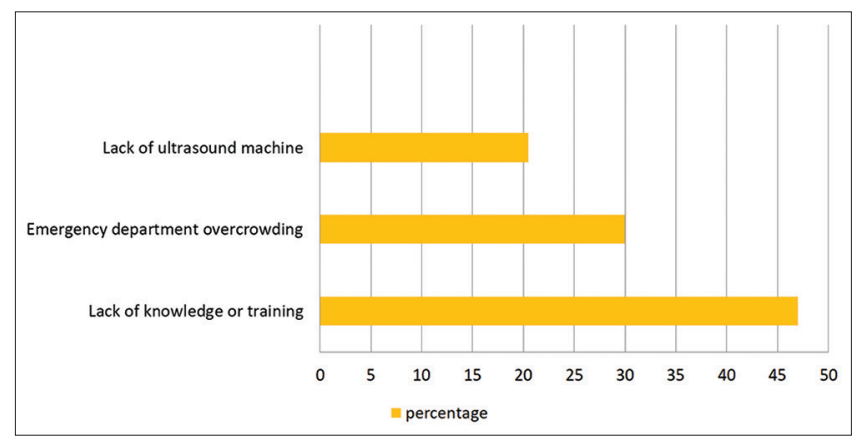

Figure 1: The barriers to perform POCUS examination

\section{Discussion}

Our results demonstrated that interns performed ultrasounds quite often which was consistent with the previous studies in general practitioners' population [6], [7], [8], [9]; however, when compared with other specialties, the rate of ultrasound use was still low [11]. In regards to the time that the ultrasound was usually performed, the morning shift was clearly the most consistent. In my opinion, this was due to the fact that we keep on a lot of staff on duty in the morning shift. Naturally, interns feel it convenient to consult staff. Our survey showed all POCUS examinations were performed for diagnostic purposes which were also consistent 
with the previous studies [12] which showed the primary aim of performing POCUS was to confirm or disconfirm a specific clinical condition (73.1\%). However, some studies [11] illustrated POCUS was used to aid physicians in performing procedureguided ultrasound. The procedures which were usually performed by ultrasound were procedural guidance for central venous access and paracentesis. Our interns were not performing ultrasoundguided procedures due to a lack of training and preparedness. In terms of the areas of examination, interns mostly performed POCUS in the abdominal area which was in contrast to previous studies which showed the area was the heart, lungs, and the inferior vena cava [13]. In terms of POCUS protocol, most interns performed only FAST examination, due to a lack of knowledge and ultrasound skills to perform other ultrasound protocols which were consistent with the medical curriculum in Thailand; that CASA, RUSH, BLUE, and other POCUS protocols were not introduced in medical students. That was in contrast with previous studies [14], [15]. In terms of the barriers experienced by interns, the results demonstrated the primary barrier was lack of knowledge or ultrasound training which was consistent with the previous studies [11] which showed the barrier was the lack of training (45.1\%). Moreover, the same barrier which demonstrated in other studies [11] was the lack of access to a POCUS machine. In our research setting, we had two ultrasound machines available in the emergency department. In addition, the number of emergency patient visits per year is nearly 70,000 visits which makes it virtually impossible to have the appropriate number of ultrasound machines.

The limitation of our study was that it was performed in a single university hospital with specific settings [16], [17], [18], [19], and [20]. Therefore, the results may not be generalized when considering other hospitals. However, our results demonstrated the obstacles of using POCUS in emergency departments that we mentioned above, which can be useful in further cases of POCUS adoption, specifically when used by interns.

\section{Conclusion}

Our findings indicated that the POCUS was typically initially performed by interns and the frequency of POCUS use was quite high. However, the administering of POCUS in the emergency department and the type of POCUS application used was limited due to lack of POCUS training which was the main obstacle. These results should be applied to the teaching curriculum for interns.

\section{Acknowledgments}

The authors would like to thank all participants in this study as well as Josh Macknick for acting as an English consultant.

\section{References}

1. Weile J, Brix J, Moellekaer AB. Is point-of-care ultrasound disruptive innovation? Formulating why POCUS is different from conventional comprehensive ultrasound. Crit Ultrasound J. 2018;10(1):25. https://doi.org/10.1186/s13089-018-0106-3 PMid:30270416

2. Steinmetz $P$, Oleskevich $S$. The benefits of doing ultrasound exams in your office. J Fam Pract. 2016;65(8):517-23.

PMid:27660835

3. Genc A, Ryk M, Suwała M, Żurakowska T, Kosiak W. Ultrasound imaging in the general practitioner's office-a literature review. J Ultrason. 2016;16(64):78-86.

PMid:27104005

4. Mengel-Jørgensen T, Jensen MB. Variation in the use of pointof-care ultrasound in general practice in various European countries. Results of a survey among experts. Eur J Gen Pract. 2016;22(4):274-7. https://doi.org/10.1080/13814788.2016.1211 105

PMid:27487159

5. Lindgaard K, Riisgaard L. Validation of ultrasound examinations performed by general practitioners. Scand J Prim Health Care. 2017;35(3):256-61. https://doi.org/10.1080/02813432.2017.135 8437

PMid:28776457

6. Bratland SZ. Ultrasonic diagnosis used in general practice. A summarized evaluation. Tidsskr Nor Laegeforen. 1985;105(28):1954-5.

PMid:3907006

7. Chan VS, Piterman L, McCall L. Use of clinical ultrasonography in an Australian suburban family practice: Its indications and findings. Hong Kong Practitioner. 1999;21(9):405-15. PMid:30670398

8. Szwamel K, Polanski P, Kurpas D. Experiences of family physicians after a CME ultrasound course. Fam Med Prim Care Rev. 2017;19(1):62-9. https://doi.org/10.5114/fmpcr.2017.66666

9. Wordsworth S, Scott A. Ultrasound scanning by general practitioners: Is it worthwhile? J Public Health Med 2002;24(2):88-94. https://doi.org/10.1093/pubmed/24.2.88 PMid:12141591

10. Olgers TJ, Azizi N, Bouma HR, Ter Maaten JC. Life after a point-of-care ultrasound course: Setting up the right conditions? Ultrasound J. 2020;12(1):43. https://doi.org/10.1186/ s13089-020-00190-7

PMid:32893335

11. Leschyna M, Hatam E, Britton S, Myslik F, Thompson D, Sedran R, et al. Current state of point-of-care ultrasound usage in Canadian emergency departments. Cureus. 2019;11(3):e4246. https://doi.org/10.7759/cureus.4246 PMid:31131169 
12. Aakjær Andersen C, Brodersen J, Davidsen AS, Graumann O, Jensen MB. Use and impact of point-of-care ultrasonography in general practice: A prospective observational study. BMJ Open. 2020;10(9):e037664. https://doi.org/10.1136/ bmjopen-2020-037664 PMid:32948563

13. lenghong K, Towsakul N, Bhudhisawasdi V, Srimookda N, Ratanachotmanee N, Phungoen P. POCUS findings in critically III patients in emergency department. J Med Assoc Thai. 2021;104:54-8.

14. Gardner KF, Clattenburg EJ, Wroe P, Singh A, Mantuani D, Nagdev A. The cardiac arrest sonographic assessment (CASA) exam-a standardized approach to the use of ultrasound in PEA. Am J Emerg Med. 2018;36:729-31. https://doi.org/10.1016/j. ajem.2017.08.052

PMid:28851499

15. Kedan I, Ciozda W, Palatinus JA, Palatinus HN, Kimchi A. Prognostic value of point-of-care ultrasound during cardiac arrest: A systematic review. Cardiovasc Ultrasound. 2020;18:1. https://doi.org/10.1186/s12947-020-0185-8

PMid:31931808
16. lenghong K, Kleebbuakwan K, Apiratwarakul K, Phungoen $\mathrm{P}$, Gaysonsiri D, Bhudhisawasdi V. Comparison of cleaning methods for ultrasound probes at an emergency department in a resource-limited country. J Med Assoc Thai. 2020;103(6):67-71.

17. Apiratwarakul $K$, Songserm $W$, lenghong $K$, Phungoen $P$, Gaysonsiri D, Bhudhisawasdi V. The role of mechanical cardiopulmonary resuscitation devices in emergency medical services. J Med Assoc Thai. 2020;103(6):98-101.

18. lenghong $\mathrm{K}$, Jumroenketpratheep $\mathrm{K}$, Tiamkao $\mathrm{S}$, Apiratwarakul $\mathrm{K}$. Use of handheld versus standard ultrasound devices in ultrasound rotation at the emergency department. Open Access Maced J Med Sci. 2021;9(E):29-32. https://doi. org/10.3889/oamjms.2021.5528

19. Apiratwarakul $K$, lenghong $K$, Gaysonsiri $D$, Mitsungnern $T$, Buranasakda M, Bhudhisawasdi V. The effectiveness of oxygen-powered inhalation devices in prehospital care. J Med Assoc Thai. 2020;103(6):58-60.

20. lenghong K, Apiratwarakul K. One year of experience in using hand-held ultrasound as a learning tool in ultrasound rotation at the emergency department. Open Access Maced J Med Sci. 2020;8(E):674-7. https://doi.org/10.3889/oamjms.2020.5573

\section{Appendix}

\section{Appendix 1: Questionnaires about participants' experiences performing POCUS examinations}

1. Your information
1.1 Sex $\square$ Male $\square$ Female
1.2 Age
year

2. The number of POCUS examinations that you performed per shift.
$\square$ 0-1 times
$\square$ 2-5 times
$\square$ 6-10 times
$\square>10$ times

3. Time of POCUS examination that you usually perform.
$\square$ Morning shift
$\square$ Afternoon shift
$\square$ Evening shift

4. Purpose of POCUS examination
$\square$ Diagnostic
$\square$ Preformed procedure

5. The area of POCUS examination that you usually perform. (you can choose more than one answers)
$\square$ Abdomen
$\square$ KUB system
$\square$ Inferior vena cava
$\square$ Heart
$\square$ Lungs
$\square$ OB-GYN
$\square$ Other

6. The POCUS protocol that you performed in this rotation. (you can choose more than one answer)

$\square$ FAST (the Focused assessment with sonography in trauma)

$\square$ CASA (the Cardiac Arrest Sonographic Assessment)

$\square$ BLUE (the Bedside lung ultrasound in emergency)

$\square$ RUSH (the Rapid Ultrasound for Shock and Hypotension)

$\square$ Other

7. The barriers to perform POCUS examination (you can choose more than one answers)
$\square$ Lack of ultrasound knowledge
$\square$ Lack of knowledge
$\square$ Emergency department overcrowding
$\square$ Other 